\section{ETIOLOGIES OF PRECOCIOUS PUBARCHE OF CHILDREN IN SOUTHERN THAILAND}

\author{
S. Jaruratanasirikul ${ }^{1}, M$. Thaiwong ${ }^{2}$ \\ ${ }^{1}$ Department of Pediatrics, ${ }^{2}$ Pediatrics, Prince of \\ Songkla University, Hat-Yai, Thailand
}

Background: Precocious pubarche is defined as the appearance of pubic hair at an age younger than 8 years in girls and 9 years in boys.

Objective: To determine the etiologies and clinical characteristics of young Thai children being evaluated for precocious appearance of pubic hair.

Subjects and method: The medical records of 32 children referred for evaluation of precocious presence of pubic hair at Songklanagarind Hospital, southern Thailand, during 1995-2009 were retrospectively reviewed.

Results: Eighty percent of the patients had pathological etiologies explaining the precocious presence of pubic hair. The most common pathological etiology was congenital adrenal hyperplasia $(50 \%)$, followed by hypothalamic hamartoma $(18.8 \%)$, adrenal tumor $(6.3 \%)$, and Leydig cell tumor (3.1\%). Only $21.8 \%$ of the patients had no pathological etiology and were diagnosed as premature adrenarche. The weight and height standard deviation scores of patients with pathological etiologies were significantly greater than those with premature adrenarche. Patients with congenital adrenal hyperplasia had significantly greater levels of basal and peak 17-hydroxyprogesterone and significantly lower levels of peak cortisol than those with premature adrenarche and adrenal tumor.

Conclusions: The presence of pubic hair in young Asian children is most commonly caused by an underlying pathological etiology. Premature adrenarche is found to be uncommon in Thai children.

\section{2-5 YEAR AGES CHILDREN AT RISK OF OVERWEIGHT BASED ON NEW WHO GROWTH STANDARDS IN AHWAZ, IRAN 2009}

S. Nouhjah ${ }^{1}$, M. Karandish ${ }^{2}$, R. Malihi ${ }^{2}$

${ }^{1}$ Public Health, School of Health Medical Sciences University of Joundishapour, ${ }^{2}$ Nutrition, Joundishapour University, Ahwaz, Iran

Background: Child hood obesity is a potentially risk factor for many health consequences, both in the short term and for their adult life. Few data of children obesity prevalence based on new WHO growth standards exist in developing countries. The aim of the study was to estimate prevalence of children at risk of overweight based on new WHO growth standards (MGRS).

Method: In a cross-sectional study 1046 children 2-5 years old (48.8\%boy, $50.2 \%$ girl) attending 18 public health centers for routine health care were surveyed. Interview with mother, filling a questionnaire by trained questioner and measurement of weight and height by trained investigators were instruments for data collection. Soft ware of WHO for PL used for data analysis after entrancing data in SPSS software. At risk of overweight was defined according to body mass index z- score (+2SD of median) using New WHO growth Standards (MGRS).

Results: Overall $15 \%$ of children were at risk of overweight (BMI/age above 2SD of median) in all age groups. This prevalence were $19.4 \%, 12.6 \%$ and $12.4 \%$ in age group $24-35,36-47,48-60$ months respectively. $16.7 \%$ of boys and $13.3 \%$ OF girl were at risk of overweight. At least prevalence was observed in 48-60 month age group of girl(8.2\%) and the most prevalence was observed in 24-35 age group of girl $(20.7 \%)$.

Conclusion: Risk of overweight in children has reached alarming proportion in Ahwaz. Urgent public health strategies are needed to prevent childhood obesity. Key words: Risk of overweight, WHO Child growth standards, 2-5 years children. 